\title{
La sostenibilidad y el papel de la contabilidad en la gestión del cambio climático y la ecoinnovación en la pyme
}

\author{
Carmen Correa Ruiz \\ Esther Albelda Pérez \\ Francisco Carrasco Fenech \\ Universidad Pablo de Olavide de Sevilla
}

\section{Resumen}

Partiendo de una reflexión sobre el papel de las pequeñas y medianas empresas (pymes) en el contexto actual de crisis para propiciar el cambio hacia una economía más sostenible, este trabajo presenta el cambio climático y la ecoinnovación como importantes retos de la sostenibilidad en las pymes. Apoyándose en una aproximación al sector vitivinícola español, se muestra el cálculo de la huella de carbono como una posible forma de ecoinnovación. También se analiza el papel de la contabilidad en el reto de la sostenibilidad y su potencial contribución para ayudar a las pymes a abordar el problema del cambio climático y mejorar la comunicación de su rendimiento ambiental.

Palabras clave: ecoinnovación, sostenibilidad, cambio climático, contabilidad, sector vitivinícola .

Código JEL: M4.

\begin{abstract}
In an attempt to reflecting on the role SMEs can play to foster change towards a more sustainable economy in the current economic crisis context, this paper portrays climate change and ecoinnovation as important challenges for SME sustainability. Grounded in the incipient experience of the Spanish wine sector, carbon footprint is shown as a way of ecoinnovation in SME. Furthermore, the paper reflects on the role of accounting for sustainability and its potential contribution to help SMEs in coping with climate change challenges and improving their environmental performance disclosure.
\end{abstract}

Keywords: ecoinnovation, sustainability, climate change, accounting, wine sector.

JEL classification: M4.

\section{Introducción}

La nueva definición de responsabilidad social de la Comisión Europea, entendida como «la responsabilidad de las empresas por su impacto en la sociedad» (CE, 2011a), ofrece oportunidades para reflexionar sobre el papel de las pequeñas y medianas empresas (pymes) en el contexto actual de crisis y su configuración como un

* Los autores quieren dejar constancia de su agradecimiento a los revisores y editores, que han contribuido sustancialmente a la mejora del trabajo. El presente trabajo ha sido parcialmente financiado por el proyecto ECO2012-33121 del Ministerio de Economía y Competitividad y el proyecto SEJ-111 de la Junta de Andalucía. 
elemento clave para propiciar el cambio hacia una economía más sostenible. En este mismo documento se insiste en la necesidad de que las empresas pongan en marcha procesos tendentes a integrar en sus operaciones y en su estrategia consideraciones sociales, medioambientales y éticas, así como aspectos relacionados con los derechos humanos e intereses de los consumidores, en estrecha colaboración con los grupos de interés, y tratando de identificar, prevenir y mitigar sus posibles efectos adversos. En este sentido, se señala la conveniencia de que las empresas adopten un enfoque a largo plazo y exploren las oportunidades para desarrollar productos y servicios innovadores así como modelos de negocio que contribuyan al bienestar de la sociedad.

Ante los desafíos económicos, sociales y ambientales a los que la sociedad se enfrenta, la Unión Europea ha realizado una apuesta decisiva por la sostenibilidad, en torno a la cual ha definido su orientación estratégica. Una pieza clave de su estrategia es la ecoinnovación, iniciativa que forma parte del programa Competencia e Innovación de la Unión Europea y que se supone que sustenta los objetivos de la Estrategia de Lisboa para la competitividad y el crecimiento económico (OCDE, 2009). Con el reclamo de ecoinnovación para un futuro sostenible, su estrategia alcanza a las pymes, a las que insta a tener en cuenta los aspectos medioambientales, para crecer de forma sostenible y mantener la competencia en el mercado global. Como señalan Blum Kusterer y Hussain (2001), la sostenibilidad es una fuerza innovadora potencialmente transformadora que permite generar nuevos productos y procesos retando y haciendo avanzar la práctica existente. Si bien muchas innovaciones sostenibles se dirigen a la mejora de procesos tecnológicos (ecoeficiencia) y a minimizar los costes de producción (Bos Brouwers, 2010), existen otras opciones disponibles, como se deriva de la propia definición de ecoinnovación de la Unión Europea.

La Unión Europea considera la ecoinnovación como cualquier cambio -tanto en productos, como procesos, métodos o estructuras organizativas- cuya finalidad suponga un significativo y demostrable avance hacia el objetivo del desarrollo sostenible, bien mediante la reducción de impactos medioambientales o bien a través de un uso más eficiente y responsable de los recursos (CE, 2006, 2010; OCDE, 2009). Incluso, destaca la utilización de diferentes etiquetas y certificaciones ambientales (tanto de productos como de procesos) como instrumentos efectivos de ecoinnovación. En particular, se ha insistido en el papel de los sistemas de gestión medioambiental para mejorar el desempeño de las organizaciones y reducir su impacto ambiental. Aguilera Caracuel y Ortiz de Mandojana (2013) señalan que el desarrollo de la ecoinnovación puede ayudar a que las empresas reduzcan sus niveles de emisión de gases de efecto invernadero (GEI). Pero también los propios procesos que permiten visualizar la contribución al cambio climático (huella de carbono) pueden constituir en sí mismos elementos de ecoinnovación.

La gestión de emisiones de gases de efecto invernadero está adquiriendo un papel cada vez más relevante, involucrando no sólo a grandes organizaciones de alto impacto ambiental sino a pequeñas y medianas empresas que supervisan y controlan sus emisiones de gases de efecto invernadero y su exposición al riesgo del cambio 
climático (PricewaterhouseCoopers, 2010) y que están empezando a calcular la huella de carbono de sus productos, servicios o incluso de toda la organización para determinar su contribución al cambio climático.

En el ámbito comunitario, se insiste en la necesidad de reforzar los vínculos entre la responsabilidad social de la empresa, la competitividad y la innovación enfatizando la importancia de la promoción de soluciones empresariales innovadoras para afrontar los actuales retos sociales y medioambientales (PE, 2013b), en la necesidad de extender la responsabilidad social a toda la cadena de suministro (PE, 2013b) así como en la necesidad de mejorar la transparencia. La Comisión Europea (CE, 2013) ha advertido de la falta de transparencia en la información no financiera y se argumenta que la actual crisis económica global se debe fundamentalmente a los problemas ocasionados por la falta de transparencia, responsabilidad, y rendición de cuentas, y la adopción de un enfoque a corto plazo (PE, 2013a). Mejorar la transparencia puede ayudar a que las empresas gestionen mejor las oportunidades y los riesgos no financieros con la consiguiente mejora del rendimiento no financiero (CE, 2013). Y, como señalan Aguilera y Ortiz (2013), es importante mejorar los mecanismos a través de los cuales las empresas comunican su rendimiento ambiental a la sociedad.

La creciente importancia de la protección del medio ambiente, la responsabilidad social, y la sostenibilidad ha abierto un nuevo mercado para la profesión contable. Muchas de las cuestiones que surgen de la búsqueda del desarrollo sostenible tienen que ver con la gestión del riesgo empresarial, un terreno de habitual preocupación e interés por parte de los contables (ICAEW, 2004). También se ha argumentado que la contabilidad puede ayudar a mejorar la visibilidad y el control interno de la gestión medioambiental, constituyéndose en un recurso intangible para fomentar el cambio organizativo hacia modelos de gestión más sostenibles (Albelda, Correa y Carrasco, 2007).

Si bien desde el ámbito académico, el debate sobre la implicación de la contabilidad en el reto medioambiental y la búsqueda del desarrollo sostenible goza de una larga tradición (Ullmann, 1976; Hines, 1991; Power, 1991; Cooper, 1992; Gray, 1992, 2002, 2010; Gray, Bebbington y Walters, 1993; Wildawsky, 1994; Lehman, 1995, 1999; Gray, Owen y Adams, 1996; Owen, Gray y Bebbington, 1997; Mathews, 1997; Neu, Warsame y Pedwell, 1998; Bebbington, Gray y Owen, 1999; Larrinaga et al., 2001, 2002; Brown y Fraser, 2006; Bebbington y Larrinaga, 2008; Parker, 2005, 2011; Correa, 2011) todavía se sigue reclamando una implicación más activa de la profesión contable a la que se reprocha el no haber asumido plenamente las implicaciones de la defensa del interés público, que constituye la esencia de la profesión (Correa, 2013).

Más concretamente, en relación con el cambio climático y las emisiones GEI, si bien se reconoce la autoridad indiscutible de la profesión contable en el terreno del suministro de información financiera relativa a derechos y obligaciones surgidas bajo los esquemas de comercio de emisiones, el reclamo de competencia en otras facetas de la contabilidad del carbono «carbon accounting» se solapa con el de otras comunidades involucradas - organizaciones científicas, firmas de consultoría, organizaciones especializadas en contabilidad de carbono, organizaciones transnaciona- 
les o nacionales de carácter gubernamental o no gubernamental- (Ascui y Lovell, 2012). No obstante, en el ámbito de la pequeña y mediana empresa, se ha señalado la responsabilidad de la profesión contable de ayudar a las empresas más pequeñas a lograr la prosperidad en el largo plazo, asesorando y proporcionando servicios que les ayuden a internalizar la sostenibilidad en su práctica empresarial y a mejorar su capacidad de aprovechar las oportunidades empresariales que brinda una economía más ecológica (FEE, 2010).

Consecuentemente, el objetivo de este trabajo es doble. En primer lugar, se pretende mostrar el cálculo de la huella de carbono como una potencial forma de ecoinnovación en la pyme en su intento de abordar el reto del cambio climático, contribuir a un desarrollo más sostenible y comunicar sus resultados ambientales a la sociedad. En segundo lugar, se pretende analizar el papel de la contabilidad en el reto de la sostenibilidad haciendo especial hincapié en su contribución para ayudar a las pymes a abordar el reto del cambio climático, y mejorar los mecanismos a través de los cuales comunican su rendimiento ambiental a la sociedad.

El trabajo se estructura en cuatro apartados. El segundo apartado reflexiona sobre el reto de la sostenibilidad en la pyme y las oportunidades que el cambio climático brinda de cara a fomentar la ecoinnovación en la pyme, presentando el cálculo de la huella de carbono como una forma de ecoinnovación que permite dar visibilidad a los resultados de la gestión ambiental y discutiendo las ventajas asociadas a su implementación así como su incipiente desarrollo y la falta de accesibilidad a dicha información. El tercer apartado, explora el incipiente desarrollo de iniciativas relacionadas con el cálculo de la huella de carbono en un sector de gran relevancia para la economía española como es el sector vitivinícola, en un intento por reflejar la difusión de dichas prácticas incluso en empresas de menor tamaño. El cuarto apartado argumenta el papel de la contabilidad en el reto de la sostenibilidad y la gestión del cambio climático, y en especial, en relación con las pymes. Finalmente, el quinto apartado presenta las conclusiones y sugiere posibles líneas de investigación futura.

\section{El reto de la sostenibilidad en las pymes: cambio climático y ecoinnovación}

Reconociendo el peso específico de las pymes en la economía española y europea, este apartado reflexiona sobre el reto de la sostenibilidad en las pymes, explorando las oportunidades que el cambio climático brinda de cara a fomentar la ecoinnovación en la pyme, y presentando el cálculo de la huella de carbono como una forma de ecoinnovación que permite dar visibilidad a los resultados de la gestión ambiental, a la vez que se generan oportunidades de mercado, mejoras en la competitividad y ahorro en costes. Se reflexiona igualmente sobre las ventajas asociadas a su implementación, sus limitaciones, así como su incipiente desarrollo y la falta de accesibilidad a dicha información.

De acuerdo con la definición de la Unión Europea, pyme es toda empresa que ocupa a menos de 250 personas y cuyo volumen de negocios anual no excede de 
50 millones de euros o cuyo balance general anual no excede de 43 millones de euros ${ }^{1}$.

Según el Ministerio de Industria, Energía y Turismo (2013), el 99,9 por 100 de las empresas españolas son pymes, porcentaje similar al de la Unión Europea (99,8 por 100). Con relación al empleo, la contribución de la pyme a la generación de empleo roza el 63 por 100 en España y algo más en la Unión Europea (67,4 por 100) (véase Cuadro 1). En términos globales, su contribución al empleo (70 por 100) y la cifra de facturación (60 por 100), es proporcional a su nivel de contaminación -el 64 por 100 de la contaminación en la Unión Europea es generado por las pymes, concretamente, entre el 60 por 100 y 70 por 100 dependiendo del sector (CE, 2010) $)^{2}$.

\section{CUADRO 1}

DISTRIBUCIÓN DE EMPRESAS Y GENERACIÓN DE EMPLEO EN ESPAÑA Y EN LA UNIÓN EUROPEA (Datos a 1 de enero de 2012)

\begin{tabular}{|c|c|c|c|c|c|c|c|}
\hline & & \multicolumn{2}{|c|}{ Micro } & \multirow{2}{*}{\begin{tabular}{|c|} 
Pequeña \\
$\begin{array}{c}(10-49) \\
\text { empleados }\end{array}$ \\
\end{tabular}} & \multirow{2}{*}{$\begin{array}{c}\text { Mediana } \\
(50-249) \\
\text { empleados }\end{array}$} & \multirow{2}{*}{\begin{tabular}{|c}
$\begin{array}{c}\text { Total } \\
\text { PYMES }\end{array}$ \\
$\begin{array}{c}(0-249) \\
\text { empleados }\end{array}$ \\
\end{tabular}} & \multirow{2}{*}{\begin{tabular}{|l} 
Grandes \\
$\begin{array}{l}\text { (250 y más) } \\
\text { empleados }\end{array}$ \\
\end{tabular}} \\
\hline & & $\begin{array}{c}0 \\
\text { empleados }\end{array}$ & $\begin{array}{c}(1-9) \\
\text { empleados }\end{array}$ & & & & \\
\hline \multirow{4}{*}{ Empresas } & \multirow{2}{*}{ España } & 1.763 .120 & 1.286 .587 & 121.601 & 20.108 & 3.191 .416 & 3.794 \\
\hline & & $55,20 \%$ & $40,30 \%$ & $3,80 \%$ & $0,60 \%$ & $99,90 \%$ & $0,10 \%$ \\
\hline & \multirow{2}{*}{$\mathbf{U E}$} & \multicolumn{2}{|c|}{19.143 .521} & 1.357 .533 & 226.573 & 20.727.627 & 43.654 \\
\hline & & \multicolumn{2}{|c|}{$92,20 \%$} & $6,50 \%$ & $1,10 \%$ & $99,80 \%$ & $0,20 \%$ \\
\hline \multirow{4}{*}{ Empleo } & \multirow{2}{*}{ España } & \multicolumn{2}{|c|}{4.076 .179} & 2.360 .300 & 2.051 .700 & 8.488.179 & 5.015 .300 \\
\hline & & \multicolumn{2}{|c|}{$30,20 \%$} & $17,50 \%$ & $15,20 \%$ & $62,90 \%$ & $37,10 \%$ \\
\hline & \multirow{2}{*}{ UE } & \multicolumn{2}{|c|}{38.395 .819} & 26.771 .287 & 22.310 .205 & 87.477.311 & 42.318 .854 \\
\hline & & \multicolumn{2}{|c|}{$29,60 \%$} & $20,60 \%$ & $17,20 \%$ & $67,40 \%$ & $32,60 \%$ \\
\hline
\end{tabular}

FUENTE: Adaptado del Ministerio de Industria, Energía y Turismo (2013) y Eurostat/National Statistic Offices of Member States.

Las pymes, por tanto, constituyen el grueso del tejido empresarial europeo y su papel en la economía de la Unión Europea es fundamental tanto desde el punto de vista económico como de creación de empleo e impacto medioambiental. Su relevancia se subraya en la Comunicación de la Comisión titulada «Pensar primero a pequeña escala «Small Business Act» para Europa: iniciativa a favor de las pequeñas empresas». En concreto, promover la innovación y facilitar que las pymes conviertan los desafíos medioambientales en oportunidades son dos de los principios básicos de la política europea (CE, 2008, 2011b), principios que podrían integrarse en un

${ }^{1}$ Recomendación 2003/361/CE de la Comisión, de 6 de mayo de 2003, sobre la definición de microempresas, pequeñas y medianas empresas [Diario Oficial L 124 de 20-5-2003].

${ }^{2}$ Datos referidos a la Unión Europea en 2006 (CE, 2010). 
solo concepto: ecoinnovación. A través de la ecoinnovación, se pretende potenciar la contribución de las pymes para lograr una economía más sostenible.

\subsection{Sostenibilidad en la pyme}

Todavía existe un vacío en la literatura sobre los procesos de innovación relacionados con la sostenibilidad en las pymes (Bos Brouwers, 2010). Durante la última década se ha empezado a generar un conocimiento más amplio sobre las particularidades de la innovación orientada a la sostenibilidad en las pymes reconociéndose cada vez más su decisiva contribución al desarrollo sostenible (Klewitz y Hansen, 2013). Como señalan Halme y Korpela (2013), en épocas de recesión y crisis económica las actividades relacionadas con la responsabilidad social suelen verse negativamente afectadas, $\mathrm{y}$ al mismo tiempo se generan amplias expectativas sobre la contribución de las pymes al desarrollo sostenible mediante soluciones innovadoras. En su estudio concluyen que existe la posibilidad de afrontar esta contradicción innovando y que las pequeñas empresas lo hacen adquiriendo y utilizando sus recursos, a menudo escasos, en la búsqueda del desarrollo sostenible. Como matiza Bos Brouwers (2010), la escasez de recursos, ya sea capital, conocimiento o mano de obra cualificada, limita seriamente la capacidad de innovación sostenible de las pymes, como también su enfoque hacia el corto plazo, que entra en contradicción con el enfoque a largo plazo que requieren las innovaciones relacionadas con la sostenibilidad. Sin embargo, y a pesar de que la falta de recursos es una barrera para las pymes, determinados tipos de innovación pueden desarrollarse de manera que esta falta de recursos se compense con una cooperación activa con clientes y otros grupos de interés (Halme y Korpela, 2013; Klewitz y Hansen, 2013). Bos Brouwers (2010), apunta que algunas pymes han tenido éxito gracias a su capacidad de orientarse a la creación de valor, a la gestión de la innovación y a la interrelación y cooperación con los grupos de interés.

\subsection{Ecoinnovación en la pyme}

Son diversas las oportunidades de negocio que la ecoinnovación actualmente puede ofrecer a las pymes -desarrollo de nuevos productos, tecnologías o servicios, mejora de los procesos productivos, o replanteamiento del modelo de negocio, algunas de las cuales, a su vez, pueden generar ahorros de costes y nuevas oportunidades de mercado así como mejoras en la competitividad (EIO y CfSD, 2013). Si bien la ecoinnovación puede estar fundamentada en avances tecnológicos, también puede estar promovida simplemente por razones medioambientales (reducción de recursos o emisiones, por ejemplo) o por meras motivaciones económicas (como la introducción de un producto ecológico), pero su característica distintiva es que genera siempre un beneficio para el medio ambiente (Arundel y Kemp, 2009; Kemp 
y Pearson, 2007). Por consiguiente, es un concepto amplio en el que tienen cabida distintas prácticas empresariales relacionadas con la integración de aspectos medioambientales tanto a nivel de productos y servicios, como de procesos, o estructuras organizativas y sistemas de gestión (Klewitz y Kansen, 2013).

Sin embargo, a pesar de la insistencia en la importancia del desarrollo de sistemas de gestión medioambiental, sólo el 0,4 por 100 de las pymes europeas tienen un sistema de gestión medioambiental certificado, bien conforme al reglamento europeo de ecogestión y ecoauditoría (EMAS), la norma ISO 14001 u otro sistema certificado a nivel nacional o sectorial (CE, 2010).

Concretamente, según el registro de EMAS, actualmente hay 3709 organizaciones registradas de las cuales 3018 son pymes (de las más de 23 millones existentes en Europa $)^{3}$. Estas herramientas que han sido creadas pensando en las grandes empresas (Bradford and Fraser, 2008; Fassin, 2008; Hillary, 2004; Jenkins, 2006), o bien en empresas con altos impactos medioambientales, resultan demasiado costosas y difíciles de implantar para pequeñas empresas sin grandes impactos. Los niveles de exigencia, los costes de auditoría y certificación externa, la burocracia y cantidad de documentación requerida, en ocasiones, hacen inviable para las pequeñas empresas su certificación. Por consiguiente, se hace necesario el desarrollo de modelos adaptados a la realidad de las pequeñas empresas, herramientas más prácticas, sencillas y que no requieran un elevado empleo de recursos.

\subsection{El cambio climático como oportunidad para la ecoinnovación y la sostenibilidad en la pyme}

No obstante, la falta de certificaciones de sistemas de gestión medioambiental no implica la ausencia de iniciativas ecoinnovadoras o de otras medidas dirigidas a reducir los impactos medioambientales. De hecho, más del 24 por 100 de las pymes europeas promueven acciones para reducir sus impactos medioambientales, aunque no tengan un sistema de gestión medioambiental certificado (CE, 2010).

La Unión Europea es consciente de la importancia de la ecoinnovación para hacer que la economía europea sea más competitiva y menos vulnerable. De hecho, la estrategia Europa 2020 se orienta hacia la búsqueda de un crecimiento inteligente, sostenible e integrador, que favorezca la transición a una economía eficiente en recursos y baja en carbono, figurando el cambio climático y la reducción de emisiones GEI entre los cinco principales objetivos de su estrategia 2020.

La gestión del cambio climático ofrece así una oportunidad a las pymes, que pueden orientar la ecoinnovación en este sentido, tratando de aprovechar los programas de incentivos que la UE ha diseñado al efecto. El primer gran impulso europeo a la ecoinnovación partió del Plan de Actuación en favor de las Tecnologías Ambientales

${ }^{3}$ Disponible en http://ec.europa.eu/environment/emas/register/reports/reports.do (consultado en agosto de 2013). 
(ETAP), adoptado en 2004, permitiendo el florecimiento de la ecoindustria europea. Como sucesor del ETAP, el Plan de Acción sobre Ecoinnovación (EcoAP), va más allá de las tecnologías ambientales para orientar la ecoinnovación hacia el fomento de una amplia gama de procesos, productos y servicios ecoinnovadores. En el marco del EcoAP (2014-2020), la principal fuente de apoyo será el programa Horizonte 2020, que tiene previsto destinar 3.160 millones de euros para la financiación de acciones relativas al clima, eficiencia de recursos y ecoinnovación. Asimismo, las pymes podrán beneficiarse de los incentivos del programa COSME orientado a mejorar la competitividad de las empresas y las pymes.

Es evidente, que el cambio climático es una de las prioridades en la agenda europea. Como piedra angular de la estrategia de la UE para reducir sus propias emisiones de gases de efecto invernadero y basado en el protocolo Kioto, se establece a principios de 2005 el régimen de comercio de derechos de emisión (EU Emissions Tranding System, EU ETS), que representa el sistema internacional más importante del mundo de límites máximos de derechos de emisión de dióxido de carbono $\left(\mathrm{CO}_{2}\right)$ y otros gases de efecto invernadero aplicado a empresas (CE, 2009).

La aprobación del protocolo de Kioto ha tenido consecuencias para las empresas españolas. La Directiva EU ETS requiere, a las instalaciones incluidas en dicho esquema, realizar un seguimiento e informar de sus emisiones de $\mathrm{CO}_{2}$. Esta expansión del mercado internacional del carbono ha favorecido la aparición de una creciente cultura de certificación y verificación en torno a las emisiones de gases de efecto invernadero ${ }^{4}$, propiciando la proliferación de empresas de consultoría y asesoramiento que ofrecen entre sus servicios los relativos a informes y verificación de emisiones.

\section{Huella de carbono como instrumento de ecoinnovación}

Sin obviar el desarrollo de la información obligatoria y la verificación relativa a la comercialización de derechos de emisión, es importante señalar el creciente interés por los informes voluntarios de gases de efecto invernadero y las mediciones de la huella de carbono tendentes a la evaluación de dichas emisiones a nivel de la organización, de la cadena de suministro y del ciclo de vida del producto.

La huella de carbono cuantifica la cantidad de gases de efecto invernadero emitidos, medidos en masa de $\mathrm{CO}_{2}$ equivalente, de una organización, evento o producto, para determinar su contribución al cambio climático. Con su cálculo se pretende identificar las fuentes de emisión GEI para poder conocer los puntos críticos de cada organización, evento o producto y así poder establecer medidas de reducción efectivas.

Son muchas las empresas que han empezado a exigir a sus proveedores datos sobre el cálculo de la huella de carbono y otras tantas las que voluntariamente han decidido calcular su huella de carbono. En esta dirección, España está preparando un

\footnotetext{
${ }^{4}$ Actualmente son siete las entidades acreditadas por la Entidad Nacional de Acreditación (ENAC) en España para poder verificar emisiones GEI.
} 
Registro Nacional de Huella de Carbono y la Oficina Española de Cambio Climático (OECC) ha anunciado la puesta en marcha en 2013 del Sistema Nacional de Huella de Carbono que estimulará a las empresas a tomar medidas para reducir sus emisiones GEI, aunque hasta la fecha la visibilidad y accesibilidad a esta información voluntaria es bastante limitada.

Existe una cierta confusión generada por la gran variedad de métodos e iniciativas desarrollados para el cálculo de la huella de carbono, algunas de ámbito nacional, local o sectorial. No obstante, si bien no existe una metodología común y uniforme, destacan algunas normas y protocolos con mayor reconocimiento internacional. Entre las metodologías más utilizadas para el cálculo de la huella de carbono de una organización sobresalen: $\mathrm{GHG}^{5}$ corporate standard, Bilan Carbon y ISO 14064; y entre las metodologías más utilizadas para el cálculo de la huella de carbono de producto: PAS $2050^{6}$, GHG product standard y ISO 14067, basadas en los principios del análisis del ciclo de vida.

La huella de carbono goza de más credibilidad si cuenta con una verificación independiente. No obstante, existen diferentes niveles de verificación: como la autodeclaración por la propia entidad, la verificación por una tercera parte experta pero no acreditada y la certificación por una tercera parte acreditada de que han seguido lo establecido en la norma o metodología de referencia elegida, siendo esta última la opción recomendable cuando se pretende comunicar a terceros la huella de carbono.

$\mathrm{Al}$ referirse a las verificaciones de huella de carbono también es importante apreciar la diversidad y alcance de las mismas. Por ejemplo, AENOR ${ }^{7}$ ofrece tres tipos de huellas de carbono: Marca AENOR Medio Ambiente de Emisiones de $\mathrm{CO}_{2}$ eqv calculadas, Marca AENOR Medio Ambiente de Emisiones de $\mathrm{CO}_{2}$ eqv compensadas, Marca AENOR Medio Ambiente de Emisiones de $\mathrm{CO}_{2}$ eqv reducidas.

Aunque el cálculo de la huella de carbono está orientado a identificar y reducir las emisiones de $\mathrm{CO}_{2}$ en productos y organizaciones para contribuir a la mitigación del cambio climático, de su implantación y utilización pueden derivarse otras ventajas tales como:

- La identificación de oportunidades de ahorro de costes.

- La contribución a la creación de un mercado de productos y servicios de bajo carbono que responda a las demandas de la sociedad y de los clientes y consumidores con conciencia ecológica.

- La demostración a terceros de la responsabilidad social de la organización y de sus compromisos con la sostenibilidad.

- La interacción de la I+D+i con otros departamentos de la organización aprovechando la sinergia y el saber hacer interno de la organización.

${ }^{5}$ GHG Protocol desarrollado por el World Business Council for Sustainable Development (WBCSD) y el World Resources Institute (WRI).

6 'PAS 2050' (Publicly Available Specification 2050:2008; 2050:2011) desarrollada por CARBON TRUST (Reino Unido).

${ }^{7}$ AENOR que es la principal empresa de certificación en España, ha realizado y concedido actualmente 41 verificaciones de la huella de carbono además de otras 15 que se encuentran en proceso. 
- El mejor posicionamiento para acceder a mercados extranjeros.

- El mejor posicionamiento para anticiparse a regulaciones futuras.

- La mejora en la credibilidad y transparencia de la información suministrada.

Sin lugar a dudas, el cambio climático puede verse como una amenaza o un reto actual, pero las organizaciones y empresas de diferentes sectores están viendo en él también una fuente de oportunidades para la mejora de su gestión interna y de sus procesos, para la racionalización de los recursos, la mejora de la eficiencia y la reducción de costes así como para la mejora de su imagen, reputación y visibilidad de su resultado ambiental, e incluso, la oportunidad de encontrar un nicho de mercado dentro de la economía baja en carbono.

\section{El sector vitivinícola y su apuesta por la huella de carbono como instrumento de ecoinnovación}

La amenaza del cambio climático es un reto para las empresas del sector vitivinícola dada su clara dependencia del medio ambiente. El incremento progresivo de las temperaturas, especialmente en los meses de verano, y la disminución de las precipitaciones inciden en el proceso de maduración de la uva y por consiguiente en la calidad de los vinos. La fijación de dióxido de carbono, el efecto cortafuegos, el valor paisajístico y cultural, el mantenimiento de la población en zonas de riesgo de abandono, la protección contra la erosión, el refugio de fauna y la preservación de especies vegetales autóctonas son algunas de las externalidades positivas derivadas del viñedo (Peris Martínez, 2013). Aparte de la evidente necesidad de gestionar el cambio climático por su especial vulnerabilidad frente al mismo, aspectos como la importancia relativa del sector en la balanza comercial, y la mejora de la competitividad de la empresa española justifican el interés de reflexionar sobre las posibilidades que el cambio climático brinda a las empresas del sector para fomentar la ecoinnovación.

El sector vitivinícola tiene una gran importancia para la economía española (1 por 100 del PIB). En primer lugar, hay que destacar que España es el primer país del mundo en superficie cultivada de viñedo ${ }^{8}$ y el tercero en producción de vino (véase Cuadro 2). Con relación a las exportaciones ${ }^{9}$, España ocupa el segundo lugar en volumen de litros $-19,66$ por 100 del total mundial-, y el tercero en términos de

${ }^{8}$ De la superficie total de viñedo en 2012, el viñedo de vinificación suponía el 98,65 por 100 del viñedo total (954.020 ha), que representa el 5,6 por 100 de la superficie de cultivo total en España (MAGRAMA, 2013).

${ }^{9}$ La industria de alimentación y bebidas es un sector estratégico en España, siendo el primer sector industrial de la economía española por volumen de ventas (7,6 por 100 del PIB español) y el segundo en exportaciones (22.078 millones de euros). El crecimiento progresivo de las exportaciones (más del 261,79 por 100 en quince años) ha contribuido a que en los dos últimos años la balanza comercial del sector sea positiva. En concreto, los principales productos exportados son la carne de cerdo y sus derivados, el vino, y el aceite de oliva. No obstante, el mayor crecimiento interanual lo ha experimentado el vino con un 16 por 100, frente al 0 por 100 del aceite de oliva (FIAB, 2013a; FIAB, 2013b). 
valor $-9,33$ por 100 del total mundial- $\left(\right.$ OIV, 2013) ${ }^{10}$. Por último, señalar que si bien las grandes empresas del sector alcanzan más de los 100 millones de euros en facturación, la mayoría de las más de 4.000 bodegas que lo conforman son cooperativas agrarias y pequeñas empresas, de tipo familiar y capital mayoritariamente de origen español (OEMV, 2013a).

\section{CUADRO 2}

SUPERFICIE CULTIVADA Y PRODUCCIÓN DE VINO EN EL MUNDO

\begin{tabular}{|l|c|c|c|c|c|c|}
\hline $\begin{array}{c}\text { Superficie } \\
\text { (miles ha) }\end{array}$ & $\mathbf{2 0 0 9}$ & $\mathbf{2 0 1 0}$ & $\mathbf{2 0 1 1}$ & Prev. 2012 & \%EU & \% mundial \\
\hline España & 1.113 & 1.082 & 1.032 & 1.018 & 29,15 & 13,44 \\
\hline Francia & 836 & 818 & 806 & 800 & 22,91 & 10,56 \\
\hline Italia & 812 & 795 & 776 & 769 & 22,02 & 10,15 \\
\hline Total UE & 3.692 & 3.619 & 3.521 & 3.492 & & 46,10 \\
\hline Total mundial & 7.702 & 7.672 & 7.592 & 7.575 & & \\
\hline $\begin{array}{c}\text { Producción } \\
\text { (miles hl) }\end{array}$ & $\mathbf{2 0 0 9}$ & $\mathbf{2 0 1 0}$ & $\mathbf{2 0 1 1}$ & Prev. 2012 & $\mathbf{\% E U}$ & \% mundial \\
\hline Francia & 46.361 & 45.704 & 50.764 & 42.243 & 29,87 & 16,80 \\
\hline Italia & 47.450 & 48.525 & 42.772 & 40.060 & 28,32 & 16,00 \\
\hline España & 35.166 & 35.235 & 33.397 & 29.665 & 20,98 & 11,80 \\
\hline Total UE & 162.898 & 156.376 & 157.171 & 141.415 & & 56,30 \\
\hline Total mundial & 271.200 & 265.100 & 266.800 & 251.000 & & \\
\hline
\end{tabular}

FUENTE: Adaptado del Informe elaborado por el Observatorio Español del Mercado del Vino (OEMV, 2013a).

Para abordar los efectos negativos del cambio climático, bien mediante estrategias de mitigación o de adaptación ${ }^{11}$, Duarte Alonso y O’Neil (2011) sugieren que tanto viticultores, bodegueros y empresas auxiliares del vino como la administración pública deben trabajar juntos, y así encontrar formas para impulsar una producción del vino más sostenible.

Al indagar sobre las cuestiones relacionadas con la sostenibilidad y el cambio climático en el sector vitivinícola, se abre un amplio abanico de posibilidades en lo que a respuesta de las empresas se refiere: esfuerzos importantes en $\mathrm{I}+\mathrm{D}+\mathrm{i}$ tendentes a mejorar la calidad y el impacto medioambiental de procesos y productos, así como a descubrir vías de diversificación -cosméticos, vinos bajos en alcohol, etc.- promo-

${ }^{10}$ No obstante, se ha exportado un volumen menor (4,5 por 100 menos respecto a 2011, 2.141,1 millones de litros) pero a mayor precio (11,6 por 100 más respecto a $2011,2.493,5$ millones de euros), a un precio medio de 1,16 euros por litro, debido principalmente a la disminución en las ventas de vino a granel y el aumento en el vino embotellado con denominación de origen (FIAB, 2013; OEMV, 2013b).

11 La primera se refiere a la minimización de las emisiones de gases de efecto invernadero; y la segunda a la capacidad de ajustar las actividades más sensibles a los cambios del clima para limitar su vulnerabilidad (IPPC, 2007). 
ción del reciclado y la reutilización, fomento de la agricultura ecológica ${ }^{12}$, construcción y edificación sostenible, estrategias de reducción de emisiones y consumos, y cálculo y reducción de la huella de carbono y de la huella hídrica, entre otras.

Dado que el transporte es una parte importante de emisión GEI del vino (Jones et al., 2005), es precisamente en la fase de envasado y transporte donde algunas bodegas han encontrado posibilidades de mejora tanto en términos de reducción de costes como de reducción del impacto medioambiental, utilizando botellas menos pesadas cuyo transporte implica un menor consumo de gasóleo y el consiguiente ahorro de costes y emisiones al transportarlas. Igualmente, con relación a la fase de envasado, el Proyecto Ecoemplea Corcho Sostenible ${ }^{13}$, también constituye una fuente de oportunidades para el sector vitivinícola, principal cliente del sector corchero.

Como consecuencia de este compromiso con la sostenibilidad y la mejora del medio ambiente en el sector del vino, entre los distintos proyectos e iniciativas que se han puesto en marcha ${ }^{14}$, destaca el manifiesto «Wineries for climate protection» promovido por la Federación Española del Vino en 2011 en Barcelona. Las bodegas y otras organizaciones relacionadas con la producción del vino que suscriben esta iniciativa pretenden con ello hacer pública su responsabilidad medioambiental dejando patente su respeto por el medio ambiente y su interés por proteger el viñedo mundial frente al cambio climático. El decálogo de las empresas firmantes incluye diez medidas para luchar contra el cambio climático ${ }^{15}$, destacando en primer lugar

${ }^{12}$ España ocupa una posición de liderazgo en la Unión Europea con una superficie ecológica de 1.845.039,10 ha en 2011 (un incremento del 115 por 100 con respecto al 2009), convirtiéndose la producción ecológica en uno de los pocos sectores de la economía que crece y genera empleo. En concreto, el cultivo de viñedo ecológico se ha incrementado un 46,4 por 100 en tan sólo dos años (79.016 ha en 2011 que representan el 12 por 100 del total de superficie ecológica cultivada). Por su parte, España es el primer productor de vino ecológico a nivel mundial, cuya producción se ha incrementado el 80,51 por 100 en dos años (161,95 miles de toneladas en 2011), al igual que el número de bodegas dedicadas a la producción de vino ecológico que también se ha incrementado (511 bodegas que representan el 16,84 por 100 de la estructura industrial del sector ecológico de origen vegetal (MAGRAMA, 2012).

${ }^{13}$ El Proyecto Ecoemplea Corcho Sostenible es una iniciativa formativa que promueve, a través de la Certificación Forestal PEFC, nuevas actitudes empresariales hacia la ecoinnovación y sostenibilidad en el monte alcornocal y en su cadena de valor en Andalucía, Castilla la Mancha, Extremadura, Cataluña y La Rioja.

${ }^{14}$ Pueden destacarse como iniciativas exclusivas del sector, entre otras:

Proyecto Cenit Deméter, que con una orientación de carácter estratégico, multidisciplinar e interregional, tras cuatro años de investigación para la adaptación al cambio climático, e involucrando a 26 empresas del sector vitivinícola, ha tratado de generar conocimientos científico-técnicos en el sector para hacer frente al cambio climático, tanto referidos a medidas de adaptación a las nuevas condiciones como para contribuir a paliar sus consecuencias.

Proyecto «Más vino, Menos $\mathrm{CO}_{2}$ » comenzó en 2010, respaldado por la Red de Desarrollo Rural (REDR) dependiente del MAGRAMA, con el objetivo de fomentar prácticas que reduzcan la emisión GEI en el sector vitivinícola. Para ello, presta ayuda a pequeñas bodegas en la realización de estudios para conocer su huella de carbono, así como impulsa la utilización de técnicas alternativas para la disminución GEI.

${ }^{15}$ Medidas propuestas relacionadas con la reducción de emisiones, edificación sostenible, energías renovables y eficiencia energética, agricultura sostenible y biodiversidad, reducción de la huella hídrica, ecodiseño, reducción de residuos, distribución eficiente, investigación e innovación, y comunicación. 
la reducción de emisiones, y comprometiéndose a reducir la huella de carbono por botella producida en la proporción fijada por la Unión Europea, es decir, 20 por 100 en el año 2020.

Además de este manifiesto, una buena parte de las iniciativas en el sector se centran más concretamente en el cálculo de la Huella de Carbono. En esta dirección, la Asociación de Empresarios Productores Ecológicos de Andalucía (EPEA), en colaboración con la Junta de Andalucía, ha puesto en marcha un proyecto para medir y verificar la huella de carbono de los productos agroalimentarios andaluces a lo largo del ciclo de vida del producto, desde la adquisición de las materias primas hasta su gestión como residuo, con la intención de que los consumidores conozcan cuál es su contribución al cambio climático y de potenciar que se implementen medidas de reducción de las emisiones GEI. A tal objeto, se ha procedido a adaptar la metodología PAS 2050 del Carbon Trust, comenzando por la implementación del proyecto en tres productos piloto: el aceite de oliva extra virgen, los tomates cherry y el vino Pedro Ximénez. El sector vitivinícola se erige como uno de los pioneros en la lucha contra el cambio climático y en el cálculo y gestión de la huella de carbono. De hecho, diversas empresas del sector están inmersas en procesos de cálculo o han calculado, certificado o verificado su huella de carbono de acuerdo con alguna de las metodologías existentes.

El primer vino del mundo en obtener la certificación de su huella de carbono por Carbon Trust según la norma PAS 2050 fue el Mobius Marlborough Sauvignon Blanc, de Nueva Zelanda, con 840 gramos de $\mathrm{CO}_{2}$ por botella. En España, el primero ha sido el Emina Verdejo 09 (D.O. Rueda) de Matarromera, con 829 gramos de $\mathrm{CO}_{2}$ por botella. Pero esto es sólo el principio de un proceso en el que parece que los viticultores y bodegueros españoles se están iniciando. El Cuadro 3 recoge algunas iniciativas sobre cálculo de huella de carbono de empresas españolas del sector. Estos datos constituyen el resultado del análisis de la información obtenida a través de una compleja búsqueda de noticias y notas de prensa, información publicada por asociaciones del sector, páginas web corporativas y de empresas consultoras o certificadoras, así como mediante solicitudes de información directamente realizadas a empresas consultoras y certificadoras. No obstante, esta información no representa un listado exhaustivo dado que, como ya se ha mencionado, no existe actualmente un registro único de huella de carbono, lo que dificulta la accesibilidad a dicha información. 


\title{
CUADRO 3
}

\section{CÁLCULO DE HUELLA DE CARBONO EN SECTOR VITIVINÍCOLA ESPAÑOL}

\author{
Huella de carbono en grandes empresas bodegueras: \\ Bodegas Campo Viejo, del grupo Domecq, se ha convertido en la primera bodega española en \\ certificar por AENOR su huella de carbono conforme a la norma ISO 14064. \\ El Grupo Faustino, se ha convertido en el primer grupo bodeguero español que ha obtenido la certifi- \\ cación de la huella de carbono de AENOR, en ocho vinos de seis bodegas de cuatro denominaciones \\ de origen (Mancha, Navarra, Ribera del Duero y Rioja): \\ - Faustino I Gran Reserva de Bodegas Faustino, D.O.Ca.Rioja (vino tinto Gran Reserva) \\ - Faustino V Reserva de Bodegas Faustino, D.O.Ca.Rioja (vino tinto Reserva) \\ - Campillo Reserva Especial de Bodegas Campillo, D.O.Ca. Rioja (vino tinto Reserva) \\ - Portia Prima de Bodegas Portia, D.O. Ribera de Duero (vino de alta expresión tinto con crianza) \\ - Triennia de Bodegas Portia, D.O. Ribera de Duero (vino de alta expresión tinto con crianza) \\ - ECCO de Bodegas Marqués de Vitoria, D.O.Ca.Rioja (tinto joven ecológico) \\ - Fortius Chardonnay de Bodegas Valcarlos, D.O. Navarra (blanco joven varietal) \\ - Finca Los Trenzones Verdejo de Bodegas Leganza, D.O. Mancha. (blanco joven varietal)
}

Anna de Codorníu, del grupo Codorniú, es el primer vino espumoso del mundo con certificación de su huella de carbono basada en la norma PAS 2050 de la entidad británica Carbon Trust.

Huella de carbono en bodegas pequeñas y medianas:

Seis bodegas riojanas han realizado el cálculo de la huella de carbono bajo la norma PAS 2050, a través de FEYDO MEDIO AMBIENTE :

- Vinícola Riojana de Alcanadre, S. COOP. Vino tinto Aradón Crianza 2010.

- Bodegas Don Sancho de Londoño. Vino tinto Cortijo 2011.

- Bodegas R. López de Heredia Viña Tondonia. Vino tinto Viña Tondonia Reserva 2002.

- Bodegas Najerilla Soc. Coop. Vino tinto Castezo Crianza 2009.

- Bodegas Puelles. Vino tinto Molino de Puelles 2009.

- Bodegas Vallemayor, S.L. Vino tinto Vallemayor Crianza 2009.

La bodega Matarromera tiene verificada por AENOR la huella de carbono de tres de sus vinos:

- Botella Emina Verdejo (añada 2009) de Bodega Matarromera

- Botella Matarromera Crianza (añada 2007) de Bodega Matarromera

- Botella Emina 12 meses (añada 2009) de Bodega Matarromera

La bodega madrileña Gosálbez-Orti (tras un estudio de la consultora Creara), ha obtenido el certificado Marca AENOR de producto Medio Ambiente $\mathrm{CO}_{2}$ Calculado para el vino Qubél Revelación 2009.

El consorcio Araex, un grupo español de bodegas familiares de la Rioja, ha alcanzado la verificación final de BSI de acuerdo a la norma PAS 2050 por el cálculo de la huella de carbono en cinco de sus vinos:

- Altos R Crianza 2009, de Altos R Bodegueros y Viticultores;

- Baigorri Crianza 2009, de Bodegas Baigorri;

- Castillo Labastida Reserva 2006, de Bodegas y Viñedos Labastida;

- Lar de Paula Reserva 2008, de Bodegas Lar de Paula

- Luis Cañas Reserva de la Familia 2006, de Bodegas Luis Cañas.

La denominación de origen Vinos de Uclés se ha convertido en la primera denominación de origen en España en certificar su huella de carbono según la ISO 14064 por OCACERT, midiendo la huella de todas sus bodegas y del consejo regulador.

La Bodega Blau Nou dispone del sello CeroCO 2012, para su vino Eccociwine, referido a sus emisiones de gases de efecto invernadero generadas por su actividad en 2011, como también obtuvo anteriormente el CeroCO 2010 y CeroCO 2011.

Actualmente está en proceso, la verificación de la huella de carbono por Bureau Veritas Certification en 14 bodegas distribuidas entre La Rioja, Aragón, Baleares, Murcia, País Vasco y Cataluña, iniciativa enmarcada dentro del proyecto «Más vino, Menos $\mathrm{CO}_{2}$ ».

FUENTE: Elaboración propia. 
La respuesta del sector vitivinícola puede obedecer a factores de índole diversa como la conciencia medioambiental, la mejora de la eficiencia energética, el ahorro en costes, o la mejora de la competitividad y de las exportaciones, pero parece evidente que ante el reto de la sostenibilidad y el cambio climático, el sector vitivinícola orienta sus esfuerzos buscando una asociación entre sostenibilidad e innovación. Y es precisamente esta asociación, la que puede favorecer la supervivencia de pymes y microempresas del sector dentro del tejido empresarial español, contribuyendo al desarrollo de una economía más sostenible.

\section{El papel de la contabilidad en el reto de la sostenibilidad y el cambio climático}

La reducción de los impactos medioambientales, la creación de valor a largo plazo y las expectativas de los grupos de interés se señalan como aspectos clave para el futuro de las empresas (UNGC, 2010) y, en este contexto, tanto desde la profesión contable (ACCA, 2012; CIMA, 2010; IFAC, 2011, 2012a, 2012b; FEE, 2010; ICAEW, 2004) como desde la investigación en contabilidad (Adams y Larrinaga, 2007; Bebbington, 2007; Gray, 2002, 2010; Owen, 2008; Parker, 2005, 2011), se ha destacado el papel de la contabilidad ante el reto de la sostenibilidad.

En un principio, la contabilidad social y medioambiental se definía como el proceso de comunicar los efectos sociales y medioambientales de las actuaciones de las organizaciones a los grupos de interés y a la sociedad en general, extendiendo los procesos de rendición de cuentas de las empresas más allá del puro suministro de información financiera a los accionistas, y asumiendo que las empresas tienen otras responsabilidades aparte de generar beneficios para sus accionistas (Gray et al., 1993). Así, una parte de la literatura se ha centrado en la información suministrada en los estados financieros sobre el resultado ambiental (Roberts, 1992; Larrinaga et al., 2002). Por otro lado, se ha prestado atención a las memorias de sostenibilidad a través de las cuales se da visibilidad al compromiso de la organización con la sostenibilidad y el desempeño alcanzado (Laine, 2005, 2009, 2010; Moneva, Archel y Correa, 2006; Milne, Tregidga y Walton, 2009). Asimismo, se ha estudiado como mediante la auditoría, el aseguramiento y la verificación se puede aportar mayor credibilidad a la información de sostenibilidad (O’Dwyer y Owen, 2005; O'Dwyer, Owen y Unerman, 2011). Más recientemente, la atención se ha centrado también en el nuevo modelo de presentación de la información (informes integrados) para proporcionar información financiera y no financiera sobre la estrategia, gobierno, comportamiento, perspectivas de futuro y sostenibilidad, mostrando las conexiones entre ellos y explicando su implicación en la creación de valor de la empresa (Correa, 2013; IIRC, 2011, 2013; Owen, 2013).

Pero el interés de la contabilidad trasciende al suministro de información y alcanza el ámbito del control y la gestión, llevando a que parte de la literatura académica se centre en analizar el papel de la contabilidad de gestión medioambiental en la mejora 
de la toma de decisiones (Bartolomeo et al., 2000; Burritt, 2004; Schaltegger y Burritt, 2000) y analizar la conexión entre el resultado ambiental y financiero (Burnett y Hassen, 2008; Henri y Journault, 2010). Más recientemente, el interés se está desviando hacia aspectos más amplios, abordando la sostenibilidad y su interrelación con los sistemas de control de gestión (Burritt, Schaltegger y Zvezdov, 2011; Durden, 2008; Gond et al., 2012; Burritt y Schaltegger, 2010; Schaltegger, Bennett, y Burritt, 2006). Sin embargo, Adams y Larrinaga (2007) y Bebbington, (2007) insisten en la necesidad de profundizar en el papel de la contabilidad de gestión ante el reto de la sostenibilidad tanto para satisfacer adecuadamente las necesidades de información (Dillard, Brown y Marshall, 2005), como para comprender mejor los procesos de cambio que contribuyen a una mayor integración de la sostenibilidad en la estrategia corporativa (Larrinaga y Bebbington, 2001; Albelda, Correa y Carrasco, 2007).

Tras la implantación de esquemas de comercio de emisiones GEI, el interés tanto de la profesión como de la investigación, se ha centrado en la valoración de los permisos de emisiones y el reflejo de los correspondientes activos y pasivos en los estados financieros (Bebbington y Larrinaga, 2008; Cook, 2009; KPMG, 2008; Lovell et al., 2010). El papel de la profesión contable en lo que a la información obligatoria relativa a la comercialización de derechos de emisión (ICAC, 2006) se refiere es indiscutible, pero la proliferación de información voluntaria de gases de efecto invernadero y las mediciones de la huella de carbono abren también un importante campo de intervención para la profesión contable aunque su competencia no sea exclusiva. En este intento de las pymes por ecoinnovar y hacer visible mediante la huella de carbono su contribución al cambio climático y los resultados de su gestión ambiental, la contabilidad puede tener un claro papel facilitador:

- ofreciendo el debido asesoramiento y ayudando a formalizar sistemas de control de gestión para que las pymes sean más ecoeficientes y sostenibles (FEE, 2010; IFAC, 2012a, 2012b),

- ayudando a mejorar la información sobre las emisiones de carbono, su medición y aseguramiento (FEE, 2009),

- contribuyendo así a dar más visibilidad a los resultados de su gestión ambiental, mejorar las decisiones y la comunicación con los distintos grupos de interés, y

- proporcionando la necesaria coordinación e integración que estas cuestiones requieren (ICAEW, 2004).

Es importante resaltar la responsabilidad que la profesión contable tiene para ayudar a las empresas más pequeñas a lograr la prosperidad en el largo plazo, poniendo su experiencia, técnicas y destrezas al servicio de las mismas para que éstas puedan aprovechar mejor las oportunidades que la ecoinnovación ofrece.

Si bien son diversas las maneras en que la contabilidad puede implicarse en el reto de la sostenibilidad, no faltan críticas a la respuesta que desde nuestra disciplina se han dado, cuestionando si las actuales prácticas de información verdaderamente reflejan el comportamiento social y ambiental de las empresas, o bien se utilizan 
para incrementar su legitimidad (Adams, 2004; Hopwood, 2009). Como Gray y Bebbington (2000, p. 8-9) señalan, la contabilidad puede ser igualmente cómplice de la degradación medioambiental, y sólo una contabilidad medioambiental crítica y autoreflexiva puede reivindicar su contribución a la sostenibilidad. Esta contabilidad, será probablemente impopular en el ámbito profesional y empresarial y, en cierto modo, sólo si resulta impopular, podríamos estar convencidos de que está empezando a remover la actual hegemonía empresarial/de los negocios.

\section{Conclusiones}

Son muchas las maneras en que las empresas pueden abordar la ecoinnovación, bien sea a través de la implantación de sistemas de gestión medioambiental, las energías renovables, la inversión en proyectos de I+D medioambiental, la reducción de la huella hídrica, la reducción y tratamiento de residuos, la orientación hacia la agricultura y producción ecológica y la construcción sostenible. El propio cálculo de la huella de carbono se muestra como una de las posibles formas de apostar por la ecoinnovación.

A través de un enfoque basado en la innovación y en la sostenibilidad, las pymes pueden brindar oportunidades de crecimiento y generación de empleo apostando por la gestión medioambiental y la economía baja en carbono y tratando de medir su contribución al cambio climático. El incipiente desarrollo actual, la diversidad de metodologías disponibles para el cálculo de la huella de carbono, la creciente cultura de certificación y verificación, las bondades y limitaciones de estas prácticas, y la manera en la que las pymes abordan el reto del cambio climático aprovechando las oportunidades que les brinda la ecoinnovación, sugieren interesantes líneas de investigación futuras.

No obstante, el cálculo y verificación de la huella de carbono es tan sólo una de las posibles respuestas de las empresas al cambio climático. No debe verse ni como un indicador absoluto de la sostenibilidad de una organización ni como una solución al problema del cambio climático, sino como una forma de ecoinnovar que puede ofrecer a las pymes, y especialmente a las microempresas, la posibilidad de desarrollar recursos y capacidades (Aragón Correa et al., 2008) para producir y prestar servicios competitivamente, contribuyendo así a mantener un cierto desarrollo socioeconómico tan necesario en momentos en que tan afectado está el tejido empresarial español. La subsistencia de las pequeñas empresas y microempresas es un factor esencial para propiciar una economía más sostenible, mucho más si los esfuerzos de éstas se orientan a tratar de subsistir y generar crecimiento y empleo apostando por la protección del entorno y la reducción de su impacto medioambiental.

Si bien no se pretende reclamar la información voluntaria sobre emisiones como un campo de competencia exclusiva de los contables, el trabajo pretende reflexionar sobre la necesaria implicación de la contabilidad en un campo que requiere una aproximación multidisciplinar. La contabilidad puede, haciendo uso de sus técnicas, 
herramientas, y experiencia, ayudar a que las pymes sean conscientes de su impacto en el entorno, en la sociedad y en la economía, difundan más claramente sus resultados ambientales y traten de mejorar su contribución al desarrollo sostenible y a la sostenibilidad del planeta.

\section{Referencias bibliográficas}

[1] ACCA (2012): Environmental Aspects of Sustainability: SMEs and the Role of the Accountant. Research report 128. The Association of Chartered Certified Accountants, Londres.

[2] ADAMS, C. (2004): «The ethical, social and environmental reporting-performance portrayal gap», Accounting, Auditing and Accountability Journal, 17(5), 731-757.

[3] ADAMS, C. y LARRINAGA GONZÁLEZ, C. (2007): «Engaging with organisations in pursuit of improved sustainability accounting and performance», Accounting, Auditing and Accountability Journal, 20(3), 333-55.

[4] AGUILERA CARACUEL, J. y ORTIZ DE MANDOJANA, N. (2013): «Green Innovation and Financial Performance: An Institutional Approach», Organization Environment, DOI: $10.1177 / 1086026613507931$.

[5] ALBELDA PÉREZ, E.; CORREA RUIZ, C. y CARRASCO FENECH, F. (2007): «Environmental management systems as an embedding mechanism: a research note», Accounting, Auditing and Accountability Journal, 20(3), 403-22.

[6] ARAGÓN CORREA, A.; HURTADO TORRES, N.; SHARMA, S. y GARCÍA MORALES, V. (2008): «Environmental strategy and performance in small firms: A resource-based perspective», Journal of Environmental Management, 86(1), 88-103.

[7] ARUNDEL, A. y KEMP, R. (2009): Measuring ecoinnovation, UNU-MERIT Working Paper 2009-017. United Nations University - Maastricht Economic and social Research and training centre on Innovation and Technology.

[8] ASCUI, F. y LOVELL, H. (2012): «Carbon accounting and the construction of competence», Journal of Cleaner Production, 36, 48-59.

[9] ASCUI, F. y LOVELL, H. (2011): «As frames collide: making sense of carbon accounting», Accounting, Auditing and Accountability Journal, 24(8), 978-999.

[10] BARTOLOMEO, M.; BENNETT, M.; BOUMA, J.; HEYDKAMP, P.; JAMES, P. y WOLTERS, T. (2000): «Environmental management accounting in Europe: current practice and future potential», European Accounting Review, 9(1), 31-52.

[11] BEBBINGTON, J. (2007): «Changing organisational attitudes and culture through sustainability accounting», en Unerman, J.; Bebbington, J. y O’Dwyer, B. (eds.), Sustainability, Accounting and Accountabiltiy, Routledge, London.

[12] BEBBINGTON, J. y LARRINAGA, C. (2008): «Carbon trading: Accounting and reporting issues», European Accounting Review, 17(4), 697-717.

[13] BEBBINGTON, J.; GRAY, R. y OWEN, D. (1999): «Seeing the wood for the trees: taking the pulse of social and environmental accounting», Accounting, Auditing and Accountability Journal, 12(1), 47-51.

[14] BLUM KUSTERER, M. y HUSSAIN, S. (2001): «Innovation and corporate sustainability: an investigation into the process of change in the pharmaceutical industry», Business Strategy and the Environment, 10, 300-316. 
[15] BOS BROUWERS, H. E. J. (2010); «Corporate sustainability and innovation in SMEs: Evidence of themes and activities in practice», Business Strategy and the Environment, 19(7), 417-435.

[16] BRADFORD, J. y FRASER, E. D. G. (2008): «Local authorities, climate change and small and medium enterprises: identifying effective policy instruments to reduce energy use and carbon emissions», Corporate Social Responsibility and Environmental Management, 15(3), 156-72.

[17] BROWN, J. y FRASER, M. (2006): «Approaches and Perspectives in Social and Environmental Accounting: an Overview of the Conceptual Landscape», Business Strategy and the Environment, 15, 103-117.

[18] BURRITT, R. (2004): «Environmental management accounting: roadblocks on the way to green and pleasant land», Business, Strategy and the Environment, 13, 13-32.

[19] BURNETT, R. y HASSEN, D. (2008): «Ecoefficiency: defining a role for environmental cost management», Accounting, Organizations and Society, 33, 551-81.

[20] BURRITT, R. y SCHALTEGGER, S. (2010): «Sustainability accounting and reporting: fad or trend?», Accounting, Auditing and Accountability Journal, 23(7), 829-46.

[21] BURRITT, R.; SCHALTEGGER, S. y ZVEZDOV, D. (2011): «Carbon management accounting: explaining practice in leading German companies», Australian Accounting Review, 21(1), 80-98.

[22] CE, Comisión Europea (2013). Recomendación de la Comisión de 9 de abril de 2013 sobre el uso de métodos comunes para medir y comunicar el comportamiento ambiental de los productos y las organizaciones a lo largo de su ciclo de vida. (2013/179/UE).

[23] CE, Comisión Europea (2011a): Communication from the Commission to the European Parliament, the Council, the European Economic and Social Committee and the Committee of the regions. A renewed EU strategy 2011-14 for corporate social responsibility. COM (2011) 681 final.

[24] CE, Comisión Europea (2011b): Communication from the Commission to the European Parliament, the Council, Economic and Social Committee and the Committee of the regions. Review of the «Small Business Act» for Europe. COM(2011) 78 final.

[25] CE, Comisión Europea (2010): SMEs and the environment in the EU -main report, prepared for the European Commission, DG Enterprise and Industry under the Competitiveness and Innovation Programme 2007-2013).

[26] CE, Comisión Europea (2009): Acción de la UE contra el cambio climático. El régimen de comercio de derechos de emisión de la UE. Comisión Europea, Dirección General de Medio Ambiente. Luxemburgo: Oficina de Publicaciones Oficiales de las Comunidades Europeas.

[27] CE, Comisión Europea (2008): Communication from the Commission to the Council, the European Parliament, the European Economic and Social Committee and the Committee of the regions «Think Small First» A «Small Business Act» for Europe. COM(2008) 394 final.

[28] CE, Comisión Europea (2006): Programa Marco para la Competitividad y la Innovación (2007-2013) (The Competitiveness and Innovation Framework Programme, CIP).

[29] CIMA (2010): Accounting for Climate Change, How accountants can help organisations mitigate and adapt to climate change. Chartered Institute of Management Accountants, Londres .

[30] COOK, A. (2009): «Emission rights: from costless activity to market operations», Accounting, Organizations and Society, 34(3-4), 456-468. 
[31] COOPER, C. (1992): «The non and nom of accounting for (M)other Nature», Accounting, Auditing and Accountability Journal, 5(3), 16-39.

[32] CORREA, C. (2013): «A Commentary on 'Integrated Reporting: A Review of Developments and their Implications for the Accounting Curriculum'», Accounting Education: An International Journal, 22(4), 360-362.

[33] CORREA, C. (2011): «Unveiling Social and Environmental Accounting Research in Spain: A Narrative of the Mobilisation of the Spanish Academic Community», Social and Environmental Accountability Journal, 31(1), 49-62.

[34] DILLARD, J.; BROWN, D. y MARSHALL, S. (2005): «An environmentally enlightened accounting», Accounting Forum, 29, 77-101.

[35] DUARTE ALONSO, A. y O’NEIL, M. (2011): «Climate change from the perspective of Spanish wine growers: a three-region study», British Food Journal, 113(2), 205221.

[36] DURDEN, C. (2008): «Towards a socially responsible management control system», Accounting, Auditing and Accountability Journal, 21(5), 671-94

[37] EIO y CfSD (2013): Ecoinnovate! A guide to ecoinnovation for SMEs and business coaches. EcoInnovation Observatory. Funded by the European Commission, DG Environment, Brussels.

[38] FASSIN, Y. (2008): «SMEs and the fallacy of formalising CSR», Business Ethics: A European Review, 17(4), 364-378.

[39] FEE (2010): Small and sustainable: opportunities for SMEs. Federation of European Accountants, Policy Statement.

[40] FEE (2009): Carbon emissions information. Federation of European Accountants, Policy Statement.

[41] FIAB (2013a): Informe Económico 2012. Federación Española de Industrias de la Alimentación y Bebidas - FIAB.

[42] FIAB (2013b): Informe Exportaciones Industria de la Alimentación y Bebidas 2012. Federación Española de Industrias de la Alimentación y Bebidas - FIAB.

[43] GOND, J.-P.; GRUBNIC, S.; HERZIG, C. y MOON, J. (2012): «Configuring management control systems: theorizing the integration of strategy and sustainability», Management Accounting Research, 23, 205-223.

[44] GRAY, R. (2010): «Is accounting for sustainability actually accounting for sustainability... and how would we know? An exploration of narratives of organisations and the planet», Accounting, Organizations and Society, 35(1), 47-62.

[45] GRAY, R. (2006): «Social, environmental and sustainability reporting and organisational value creation. Whose value? Whose creation?», Accounting, Auditing \& Accountability Journal, 19(6), 793-819.

[46] GRAY, R. (2002): «The social accounting project and accounting organisations and society: privileging engagement, imaginings, new accountings and pragmatism over critique?», Accounting, Organisations and Society, 27(7), 687-708.

[47] GRAY, R. (1992): «Accounting and environmentalism: an exploration of the challenge of gently accounting for accountability, transparency and sustainability», Accounting, Organizations and Society, 17(5), 399-426.

[48] GRAY, R. y BEBBINGTON, J. (2000): «Environmental accounting: managerialism and sustainability: is the planet safe in the hands of business and accounting?», Advances in Environmental Accounting, Organizations and Management, 1, 1-44. 
[49] GRAY, R.; BEBBINGTON, J y WALTERS, D. (1993): Accounting and the Environment: The greening of accountancy, part II. Paul Chapman Publishing: London.

[50] GRAY, R.; OWEN, D. y ADAMS, C. (1996): Accounting and accountability: Changes and challenges in corporate social and environmental reporting. London: Prentice Hall.

[51] HALME, M. y KORPELA, M. (2013): «Responsible innovation toward sustainable development in small and medium-sized enterprises: a resource perspective», Business Strategy and the Environment. DOI: 10.1002/bse.1801.

[52] HENRI, J. F. y JOURNAULT, M. (2010): «Ecocontrol: the influence of management control systems on environmental and economic performance», Accounting, Organizations and Society, 35, 63-80.

[53] HILLARY, R. (2004): «Environmental management systems and the smaller enterprise», Journal of Cleaner Production, 12(6), 561-569.

[54] HINES, R. D. (1991): «Accounting for Nature», Accounting, Auditing and Accountability Journal, 4(3), 27-29.

[55] HOPWOOD, A. G. (2009): «Accounting and the environment», Accounting, Organizations and Society, 34(3-4), 433-439.

[56] ICAC (2006): Resolución de 8 de febrero de 2006, del Instituto de Contabilidad y Auditoría de Cuentas, por la que se aprueban normas para el registro, valoración e información de los derechos de emisión de gases de efecto invernadero.

[57] ICAEW (2004). Information for Better Markets Sustainability: the role of accountants. An initiative from the Institute of Chartered Accountants in England \& Wales.

[58] IFAC (2012a): The role of SPMs in greening small business, International Federation of Accountants, Small and Medium Practices Committee, International Federation of Accountants, New York.

[59] IFAC (2012b): Small and Medium Practices Committee, the Role of SMPs in Greening Small Business. International Federation of Accountants, New York.

[60] IFAC (2011): Sustainability Framework 2.0. Professional Accountants as Integrators, International Federation of Accountants, New York, NY.

[61] IIRC (2013): Draft International Integrated Reporting Framework. Integrated Reporting Council -IIRC.

[62] IIRC (2011): The Discussion Paper Towards Integrated Reporting - Communicating Value in the 21 st Century. Integrated Reporting Council -IIRC.

[63] KPMG (2008): Accounting for carbon: The impact of carbon trading on financial statements. KPMG LLP(UK), London, UK.

[64] JENKINS, H. (2006): «Small business champions for corporate social responsibility», Journal of Business Ethics, 67(3), 241-56.

[65] JONES, G. V.; WHITE, M. A.; COOPER, O. R. y STORCHMANN, K. (2005): «Climate change and global wine quality», Climatic Change, 73(3), 319-343.

[66] KEMP, R. y PEARSON (2007): Final report MEI project about measuring ecoinnovation. Disponible en: http://www.oecd.org/env/consumption-innovation/43960830.pdf

[67] KLEWITZ, J. y HANSEN, E. (2013): «Sustainability-oriented innovation of SMEs: a systematic review», Journal of Cleaner Production, DOI: 10.1016/j.jclepro.2013.07.017.

[68] LAINE, M. (2005): «Meanings of the term 'Sustainable Development' in Finnish corporate disclosures», Accounting Forum, 29, 395-413. 
[69] LAINE, M. (2009): «Ensuring legitimacy through rhetorical changes? A longitudinal interpretation of the environmental disclosures of a leading Finnish chemical company», Accounting, Auditing and Accountability Journal, 22(7), 1029-1054.

[70] LAINE, M. (2010): «Towards sustaining the status quo: Business talk of sustainability in Finnish corporate disclosures 1987-2005», European Accounting Review, 19(2), 247 274.

[71] LARRINAGA GONZALEZ, C. y BEBBINGTON, J. (2001): «Accounting change or institutional appropriation? A case study of the implementation of environmental accounting», Critical Perspectives on Accounting, 12, 269-92.

[72] LARRINAGA, C.; CARRASCO, F.; CARO, F. J.; CORREA, C. y PÁEZ, J. M. (2001): «The role of environmental accounting in organizational change: an exploration of Spanish companies», Accounting, Auditing and Accountability Journal, 14(2), 213-39.

[73] LARRINAGA, C.; MONEVA, J. M.; LlENA, F.; CARRASCO, F. y CORREA, C. (2002): Regulación contable de la información medioambiental. Normativa española e internacional. AECA: Madrid.

[74] LEHMAN, G. (1999): «Disclosing new worlds: a role for social and environmental accounting and auditing», Accounting, Organizations and Society, 24(3), 217-242.

[75] LEHMAN, G. (1995): «A legitimate concern for environmental accounting», Critical Perspectives on Accounting, 6(6), 393-412.

[76] LOVELL, H.; DE AGUIAR, T. S.; BEBBINGTON, J. y LARRINAGA-GONZÁLEZ, C. (2010): Accounting for Carbon. The Association of Chartered Certified Accountants, London, UK.

[77] MAGRAMA (2013): Encuesta sobre Superficies y Rendimientos de Cultivos -ESYRCE 2012-. Análisis de las Plantaciones de Viñedo en España. Ministerio de Agricultura, Alimentación y Medio Ambiente - MAGRAMA.

[78] MAGRAMA (2012): Caracterización del sector de la producción ecológica española en términos de valor, volumen y mercado. Ministerio de Agricultura, Alimentación y Medio Ambiente - MAGRAMA.

[79] MATHEWS, M.R. (1997): «Twenty-five years of social and environmental accounting: is there a silver jubilee to celebrate?», Accounting, Auditing and Accountability Journal, 10(4), 481-531.

[80] MINISTERIO DE INDUSTRIA, ENERGÍA Y TURISMO (2013): Retrato de las PYME 2013. Dirección General de Industria y de la PYME. Subdirección General de Apoyo a la PYME.

[81] MILNE, M.; TREGIDGA, H. y WALTON, S. (2009): «Words not actions!: The ideological role of sustainable development reporting», Accounting, Auditing and Accountability Journal, 22(8), 1211-1257.

[82] MONEVA, J. M.; ARCHEL, P. y CORREA, C. (2006): «GRI and the camouflaging of corporate unsustainability», Accounting Forum, 30, 121-137.

[83] NEU, D.; WARSAME, H. y PEDWELL, K. (1998): «Managing public impresions: environmental disclosures in annual reports», Accounting, Organizations and Society, 23(3), 265-282.

[84] OCDE (2009): Sustainable Manufacturing and EcoInnovation, Synthesis Report.

[85] OIV (2013): Situación mundial de la vitivinicultura en 2012. Presentación realizada por el director general de la Organización Internacional del Vino en el XXXV Congreso mundial de la vid y el vino celebrada en Bucarest (3 de junio de 2013). 
[86] OEMV (2013a): El Vino en cifras - Año 2012. Informe elaborado por el Observatorio Español del Mercado del Vino - OEMV.

[87] OEMV (2013b): Exportaciones españolas de vino, año 2012. Datos definitivos.

[88] O’Dwyer, B. y Owen, D. (2005): «Assurance statement practice in environmental, social and sustainability reporting: a critical evaluation», The British Accounting Review, 37, 205-229.

[89] O’DWYER, B.; OWEN, D. y UNERMAN, J. (2011): «Seeking legitimacy for new assurance forms: The case of assurance on sustainability reporting», Accounting, Organizations and Society, Vol. 36 No 1, pp. 31-52.

[90] OWEN, G. (2013): «Integrated Reporting: A Review of Developments and their Implications for the Accounting Curriculum», Accounting Education: An International Journal, 22(4), 340-356.

[91] OWEN, D. (2008): «Chronicles of wasted time? A personal reflection on the current state of, and future prospects for, social and environmental accounting research», $A c$ counting, Auditing and Accountability Journal, 21(2), 240-67.

[92] OWEN, D.; GRAY, R. y BEBBINGTON, K. J. (1997): «Green accounting: cosmetic irrelevance or radical agenda for change», Asia-Pacific Journal of Accounting, 4(2), 175-198.

[93] PARKER, L. (2011): «Twenty-one years of social and environmental accountability research: A coming of age», Accounting Forum, 35(1), 1-10.

[94] PARKER, L. (2005): «Social and environmental accountability research: A view from the commentary box», Accounting, Auditing and Accountability Journal, 18(6), 842 860.

[95] PE, Parlamento Europeo (2013a): Directiva 2013/34/UE del Parlamento Europeo y del Consejo de 26 de junio de 2013 sobre los estados financieros anuales, los estados financieros consolidados y otros informes afines de ciertos tipos de empresas, por la que se modifica la Directiva 2006/43/CE del Parlamento Europeo y del Consejo y se derogan las Directivas 78/660/CEE y 83/349/CEE del Consejo.

[96] PE, Parlamento Europeo (2013b): European Parliament Report on corporate social responsibility: accountable, transparent and responsible business behaviour and sustainable growth, A7-0017/2013.

[97] PERIS MARTÍNEZ, B. (2013): «Valoración ambiental de viñedos mediante métodos multicriterio. Aplicación a la valoración del viñedo del término municipal de Requena», Revista digital Ojeando la Agenda, 22, p. 3-29.

[98] POWER, M. (1991): «Auditing and environmental expertise: between protest and professionalization», Accounting, Auditing and Accountability Journal, 4(3), 30-42.

[99] PRICEWATERHOUSECOOPERS (2010): Carbon disclosure project 2010: global 500 report. PricewaterhouseCoopers, Sidney, Australia.

[100] ROBERTS, R. W. (1992): «Determinants of corporate social responsibility disclosure: An application of stakeholder theory», Accounting, Organizations and Society, 17 (6), 595-612.

[101] SCHALTEGGER, S. y BURRITT, R. (2000): Contemporary environmental accounting: issues, concepts and practice. Greenleaf, Sheffield.

[102] SCHALTEGGER, S.; BENNETT, M. y BURRITT, R. (2006): Sustainability Accounting and Reporting. Springer, Dordrecht, The Netherlands. 
[103] ULLMANN,A. E. (1976): «The corporate environmental accounting system: a management tool for fighting environmental degradation», Accounting, Organizations and Society, 1(1), 71-79.

[104] UNGC, United Nations Global Compact (2010): A New Era of Sustainability. CEO Reflections on Progress to Date, Challenges Ahead and the Impact of the Journey toward a Sustainable Economy, Study 10, UN Global Compact-Accenture, New York, NY.

[105] WILDAVSKY, A. (1994): «Accounting for the environment», Accounting, Organizations and Society, 19(4-5). 